\title{
Genetic engineering of Escherichia coli to improve L-phenylalanine production
}

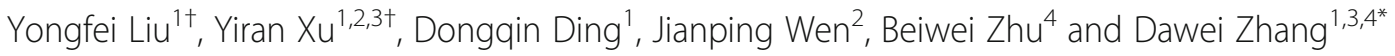

\begin{abstract}
Background: L-phenylalanine (L-Phe) is an essential amino acid for mammals and applications expand into human health and nutritional products. In this study, a system level engineering was conducted to enhance L-Phe biosynthesis in Escherichia coli.

Results: We inactivated the PTS system and recruited glucose uptake via combinatorial modulation of galP and glk to increase PEP supply in the XIlp01 strain. In addition, the HTH domain of the transcription factor TyrR was engineered to decrease the repression on the transcriptional levels of L-Phe pathway enzymes. Finally, proteomics analysis demonstrated the third step of the SHIK pathway (catalyzed via AroD) as the rate-limiting step for L-Phe production. After optimization of the aroD promoter strength, the titer of L-Phe increased by $13.3 \%$. Analysis of the transcriptional level of genes involved in the central metabolic pathways and L-Phe biosynthesis via RT-PCR showed that the recombinant L-Phe producer exhibited a great capability in the glucose utilization and precursor (PEP and E4P) generation. Via systems level engineering, the L-Phe titer of XIlp21 strain reached $72.9 \mathrm{~g} / \mathrm{L}$ in a $5 \mathrm{~L}$ fermenter under the non-optimized fermentation conditions, which was 1.62-times that of the original strain XIIp01.
\end{abstract}

Conclusion: The metabolic engineering strategy reported here can be broadly employed for developing genetically defined organisms for the efficient production of other aromatic amino acids and derived compounds.

Keywords: L-phenylalanine, Metabolic engineering, Proteomics, TyrR, AroD

\section{Background}

L-Phenylalanine is an essential and commercially available aromatic amino acid that has been widely used in pharmaceuticals and as food additive [1,2]. More than 30,000 tons of L-Phe are needed annually to meet the increasing demand for the low-calorie sweetener aspartame [3]. Moreover, L-Phe is extensively used in pharmaceutically active compounds, such as anti-inflammatory drugs [4], central nervous system neuropeptides, and HIV protease inhibitors [2]. Due to the rapid development of metabolic engineering, it is possible to rationally design the genetic circuits thus producing L-Phe in microorganisms $[5,6]$. In fast-growing bacteria such as Escherichia coli or Corynebacterium glutamicum, high titers of L-Phe $(57 \mathrm{~g} / \mathrm{L}$ and

\footnotetext{
* Correspondence: zhang_dw@tib.cas.cn

${ }^{\dagger}$ Equal contributors

${ }^{1} T$ ianjin Institutes of Industrial Biotechnology, Chinese Academy of Sciences, Tianjin 300308, People's Republic of China

${ }^{3}$ Key Laboratory of Systems Microbial Biotechnology, Chinese Academy of Sciences, Tianjin 300308, People's Republic of China

Full list of author information is available at the end of the article
}

$50 \mathrm{~g} / \mathrm{L}$, respectively) can be achieved via metabolic network optimization $[7,8]$.

In E. coli, the L-Phe synthetic pathway has two precursors: phosphoenolpyruvate (PEP) and erythrose-4phosphate (E4P), which were generated from the Embden-Meyerhof-Parnas (EMP) pathway and pentose phosphate (PP) pathway, respectively. However, more than $50 \%$ of PEP was used for the translocation and phosphorylation of glucose by the phosphotransferase system (PTS) [3]. Replacement of this glucose transport and phosphorylation capabilities of the PTS would be an efficient approach for high PEP availability. Chandran et al. [9] used glucose facilitator and glucokinase ( $g l f$ and $g l k$ ) from Zymomonas mobilis for glucose uptake in E. coli, thus greatly improving the production of shikimic acid. Meza et al. overexpressed the D-galactose transporter GalP in PTS inactivated E. coli and increased the yield of aromatics 10-times [10].

The condensations of PEP and E4P were catalyzed via 3-deoxy-D-arabinoheptulosonate-7-phosphate (DAHP) synthase [10]. This synthase contains three isoforms: 
AroG, AroH, and AroF, which are subject to transcriptional and allosteric feedback-regulation by L-Phe, Ltryptophan (L-Trp), and L-tyrosine (L-Tyr), respectively. Another bottleneck in the L-Phe synthetic pathway appears in the formation of phenylpyruvate, catalyzed by the bifunctional enzyme chorismate mutase/prephenate dehydratase (PheA). PheA plays a significant role in phynylpyruvate production and this enzyme is feedback inhibited via allosteric binding of L-Phe. To improve the production of L-Phe in E. coli, much work has been devoted to deregulating the feedback inhibition of DAHP synthase and PheA to channel the carbon influx from the central metabolism towards L-Phe [3]. Ray et al. [11] found AroH to be insensitive to Trp by replacing Val ${ }^{147}$ to $\mathrm{Gly}^{149}$. Tyr-insensitive AroF mutants were obtained by replacing Pro ${ }^{148}$ or $\mathrm{Gln}^{152}$ or deletion of $\mathrm{Ile}^{11}$. Zhou et al. [12] selected a L-Phe producer with PheAmut (T326P) from the N-methyl- $\mathrm{N}^{\prime}$-nitro-N-nitrosoguanidine (NTG) mutation library and reported that PheA (T326P) exerted a maximum L-Phe titer of $57.63 \mathrm{~g} / \mathrm{L}$ with high L-Phe productivity $(1.5 \mathrm{~g} / \mathrm{L} / \mathrm{h})$, which was the highest level reported so far. Zhou et al. [8] overexpressed $p h e A^{f b r}$ as well as wild-type $a r o F^{w t}$ in the E. coli strain WSH-Z06 and the L-Phe titer of the recombinant strain reached $35.38 \mathrm{~g} / \mathrm{L}$ in a $3 \mathrm{~L}$ fermenter, which was 2.81-times higher than the original strain and the L-Phe yield on glucose $(0.26 \mathrm{~mol} / \mathrm{mol})$ was two-times higher than that of the initial strain.

The TyrR regulator of $E$. coli is a class I transcription factor. This regulator constitutes more than nine transcription units, each of which is regulated in a distinctive manner by TyrR [13]. TyrR has three functional domains: the $\mathrm{N}$-terminal domain, the central domain, and the C-terminal domain [14]. The N-terminal domain is able to bind aromatic amino acid ligands and interact with the $\alpha$-subunit of RNA polymerase to activate the transcription of specific genes. The central domain contains an ATP binding domain, which is able to strengthen the affinity between the TyrR protein and the DNA recognition site in the $\mathrm{C}$-terminal domain by nearly 4-times. The $\mathrm{C}$-terminal domain contains $\alpha$ helixturn-helix (HTH) motif and is responsible for binding to the TyrR box sequence of controlled genes.

The TyrR regulon consists of more than nine transcription units and the regulation mechanisms vary between each individual unit $[14,15]$. Up to now, 13 TyrR regulons (including TyrR itself) have been identified by using the method of genomic SELEX (9). These regluons include: AroF, AroL TyrP, Mtr, TyrB, AroP, AroG CusC, CyaA, ProP, FolA, and HolE [15]. These mechanisms were discovered by observing changes of the transcription level of particular genes in either presence or absence of aromatic amino acids and were further validated by observing the results of activation or repression after introducing site mutations into the TyrR [16]. Mutations in the ATP-binding domain affect the Phe-mediated and Tyr-mediated repression of the aroG gene [16].

In light of the discussion above, system level engineering was conducted in the L-Phe producer Xllp01. Firstly, we inactivated the PTS system and then cooverexpressed galP and glk genes to decrease consumption of PEP. In addition, due to the lack of relevant research on the engineering transcription factor TyrR in the industrial $E$. coli strains, we attempted to engineer the HTH domain of the TyrR to investigate the effects of the TyrR mutants on the transcription level of genes involved in the L-Phe biosynthetic pathway. Furthermore, proteomics technology was applied to provide a global protein expression profile of strains by comparing the quantities of proteins between the E. coli wild type and the engineered L-Phe producer.

\section{Methods \\ Bacterial strains and cultivation conditions}

All strains and plasmids used in this study are listed in Table 1. The xllp01 strain was renamed in this experiment and the genome background of the strain was the same as the HD-1 strain in the publication [17].

The culture media used in this study included LuriaBertani (LB) liquid culture medium $(10 \mathrm{~g} / \mathrm{L} \mathrm{NaCl}, 5 \mathrm{~g} / \mathrm{L}$ yeast extract, and $10 \mathrm{~g} / \mathrm{L}$ Tryptone), M9 media broth $\left(1 \mathrm{~g} / \mathrm{L} \mathrm{NH} \mathrm{NH}_{4} \mathrm{Cl}, 6.5 \mathrm{~g} / \mathrm{L} \mathrm{Na}_{2} \mathrm{HPO}_{4}, 3.5 \mathrm{~g} / \mathrm{L} \mathrm{KH}_{2} \mathrm{PO}_{4}, 40 \mathrm{~g} /\right.$ L glucose, $0.24 \mathrm{~g} / \mathrm{L} \mathrm{MgSO}_{4}$, and $0.01 \mathrm{~g} / \mathrm{L} \mathrm{CaCl}_{2}$ ), and recovery broth $(20 \mathrm{~g} / \mathrm{L}$ Tryptone, $5 \mathrm{~g} / \mathrm{L}$ Yeast Extract, $0.5 \mathrm{~g} / \mathrm{L} \mathrm{NaCl}, 0.19 \mathrm{~g} / \mathrm{L} \mathrm{KCl}, 0.95 \mathrm{~g} / \mathrm{L} \mathrm{MgCl}_{2}$, and $3.6 \mathrm{~g} / \mathrm{L}$ glucose).

For shake flask fermentation, a single colony was inoculated into $5 \mathrm{~mL}$ of LB medium with appropriate antibiotics and cultured overnight at $37{ }^{\circ} \mathrm{C}$. The strains were then inoculated into a $500 \mathrm{~mL}$ shake flask containing $20 \mathrm{~mL}$ of seed culture medium $(8 \mathrm{~g} / \mathrm{L}$ yeast extract, $14 \mathrm{~g} / \mathrm{L}\left(\mathrm{NH}_{4}\right)_{2} \mathrm{SO}_{4}, 2 \mathrm{~g} / \mathrm{L}$ sodium citrate, $4 \mathrm{~g} / \mathrm{L} \mathrm{KH}_{2} \mathrm{PO}_{4}$, $20 \mathrm{~g} / \mathrm{L}$ glucose, $8 \mathrm{mg} / \mathrm{L} \mathrm{FeSO}_{4} \cdot 7 \mathrm{H}_{2} \mathrm{O}, 40 \mathrm{mg} / \mathrm{L}$ thiamine, and $2 \mathrm{~g} / \mathrm{L} \mathrm{MgSO}_{4}$ ) at a ratio of 1:100 and incubated at $37^{\circ} \mathrm{C}, 220 \mathrm{rpm}$ for $8 \mathrm{~h}$. After this, the seed culture was transferred into the fermentation medium $(20 \mathrm{~g} / \mathrm{L}$ glucose, $10 \mathrm{~g} / \mathrm{L}\left(\mathrm{NH}_{4}\right)_{2} \mathrm{SO}_{4}, 5 \mathrm{~g} / \mathrm{L} \mathrm{KH}_{2} \mathrm{PO}_{4}, 5 \mathrm{~g} / \mathrm{L} \mathrm{MgSO}_{4}$, $4 \mathrm{~g} / \mathrm{L}$ yeast extract, $0.015 \mathrm{~g} / \mathrm{L} \mathrm{FeSO}_{4} \cdot 7 \mathrm{H}_{2} \mathrm{O}, 0.015 \mathrm{~g} / \mathrm{L}$ $\mathrm{MnSO}_{4} \cdot \mathrm{H}_{2} \mathrm{O}, 3 \mathrm{~g} / \mathrm{L}$ betaine, $10 \mu \mathrm{g} / \mathrm{L}$ biotin, and $250 \mathrm{mg} /$ $\mathrm{L}$ Tyr) at a ratio of 1:30. The corresponding antibiotics were added into the culture medium. The cultivation was performed in triplicate for each strain.

To cultivate strains in a $5 \mathrm{~L}$ fermenter, strains that were cultivated in LB overnight were inoculated into the seed culture for $7 \mathrm{~h}$ at $37^{\circ} \mathrm{C}$ and then inoculated into a $5 \mathrm{~L}$ fermenter containing $3.5 \mathrm{~L}$ seed culture medium $(1: 10, v / v)$. The $\mathrm{pH}$ was controlled at $7.0 \pm 0.1$ via 
Table 1 Strains and plasmids used in this study

\begin{tabular}{|c|c|c|}
\hline Strains and plasmid & Genotype & Source or reference \\
\hline E. coli W3110 & $\begin{array}{l}\text { Wild type } \\
\text { W3110 derived mutant L-tyrosineauxotrophic }\end{array}$ & Our lab \\
\hline XIlp01 & $\begin{array}{l}\text { p15A:::pheA-Thr326Pro } \\
\text { p15A::aroF carried by PL promoter kan }{ }^{r}\end{array}$ & Our lab \\
\hline XIlp02 & Xllp01 $\Delta p t s H$ & This work \\
\hline XIlp03 & XIlp02 Pm37-galp Pm37-glk & This work \\
\hline XIlp04 & XIlp02 Pm37-galp Pm93-glk & This work \\
\hline Xllp05 & XIlp02 Pm93-galp Pm37-glk & This work \\
\hline XIlp06 & XIlp02 Pm93-galp Pm93-glk & This work \\
\hline XIlp07 & Xllp04 tyrR S493 T & This work \\
\hline XIlp08 & Xllp04 tyrR T495I & This work \\
\hline XIlp09 & Xllp04 tyrR N499D & This work \\
\hline XIlp10 & XIlp04 tyrR A498V & This work \\
\hline Xllp11 & Xllp04 tyrR S482 N & This work \\
\hline XIlp12 & XIlp08 pBR322:: tktA carried by trc promoter Ampr & This work \\
\hline Xllp13 & XIlp08 pBR322:: ppsA carried by trc promoter Ampr & This work \\
\hline XIlp14 & XIlp08 pBR322:: pckA carried by trc promoter Amp ${ }^{r}$ & This work \\
\hline XIlp15 & XIIp08 pBR322:: talB carried by trc promoter Amp ${ }^{r}$ & This work \\
\hline XIlp16 & XIlp08 pBR322:: aroD carried by trc promoter Amp ${ }^{r}$ & This work \\
\hline XIlp17 & XIlp08 pBR322:: ydiB carried by trc promoter Ampr & This work \\
\hline XIlp18 & XIlp08 pBR322:: aroC carried by trc promoter $\mathrm{Amp}^{r}$ & This work \\
\hline Xllp19 & XIlp08 pBR322:: aroD carried by BBa_j23109 promoter Ampr & This work \\
\hline XIlp20 & XIlp08 pBR322:: aroD carried by BBa_j23105 promoter Amp ${ }^{r}$ & This work \\
\hline XIlp21 & XIlp08 pBR322:: aroD carried by BBa_j23106 promoter Ampr & This work \\
\hline XIlp22 & XIlp08 pBR322:: aroD carried by BBa_j23101 promoter Amp ${ }^{r}$ & This work \\
\hline XIlp23 & XIlp08 pBR322:: aroD carried by BBa_j23100 promoter Ampr & This work \\
\hline $\mathrm{p}-t k t A$ & pBR322::tktA trc promoter Ampr & This work \\
\hline p-ppsA & pBR322::ppsaA trc promoter Amp ${ }^{r}$ & This work \\
\hline $\mathrm{p}$-talB & pBR322::talB trc promoter Ampr & This work \\
\hline$p-p c k A$ & pBR322::pckA trc promoter Ampr & This work \\
\hline$p$-aroD & pBR322::aroD trc promoter $\mathrm{Amp}^{\mathrm{r}}$ & This work \\
\hline p-ydiB & pBR322::ydiB trc promoter Amp ${ }^{r}$ & This work \\
\hline p-aroc & pBR322::aroC trc promoter Amp ${ }^{r}$ & This work \\
\hline p-j23100-arod & pBR322::aroD BBa_j23100 promoter Ampr & This work \\
\hline p-j23101-arod & pBR322::aroD BBa_j23101 promoter Ampr & This work \\
\hline p-j23106-arod & pBR322::aroD BBa_j23106 promoter Amp ${ }^{r}$ & This work \\
\hline p-j23105-arod & pBR322::aroD BBa_j23105 promoter Amp ${ }^{r}$ & This work \\
\hline p-j23109-arod & pBR322::aroD BBa_j23109 promoter Ampr & This work \\
\hline pKD46 & applied for gene knocking-out & {$[18]$} \\
\hline pDS132 & applied for gene knocking-out & [19] \\
\hline P15A & applied for gene overexpression & {$[26]$} \\
\hline
\end{tabular}

automatic addition of a $25 \%$ ammonia water, and the dissolved oxygen (DO) was constantly retained at $40 \%$ saturation by cascading the agitation speed (400$1000 \mathrm{rpm})$ and aeration rate (2-5 vvm). Glucose feeding was performed with a peristaltic pump when the initial glucose in the medium was exhausted and the concentration of glucose maintained below $5 \mathrm{~g} / \mathrm{L}$. Samples were collected every $2 \mathrm{~h}$ to determine cell density $\left(\mathrm{OD}_{600}\right)$, 
residual glucose, off-line $\mathrm{pH}$, amino acid concentrations (after $16 \mathrm{~h}$ ), and plasmid stability.

\section{Amplification and overexpression genes}

For gene overexpression, target genes were amplified from the genomic DNA of Xllp01 and inserted into the pBR322 plasmid. PCR was employed to introduce endonuclease restriction sites at the end of the gene fragments by using the primers shown in Additional file 1: Table S1. Genomic editing described by Datsenko and Wanner [18] was used to obtain E. coli derived strains in this research. The sacB-flanked chloramphenicolresistance gene was amplified from plasmid pDS132 and the upstream and downstream homology of target genes were amplified from W3110 genome. Then the three fragments were then fused together by fusion PCR [19]. Site mutations on TyrR were achieved by using the technique of rapid PCR site-directed mutagenesis in vitro (KOD-Plus-Mutagenesis Kit, TOYOBO). Fragments were transformed into E. coli via electrotransformation.

\section{Analysis of L-Phe production using HPLC}

L-Phe titers were measured using high-performance liquid chromatography (HPLC) with UV detection. Samples collected from either shake flasks or $5 \mathrm{~L}$ fermenter were centrifuged and the supernatants were diluted with the required amount of $d d \mathrm{H}_{2} \mathrm{O}$. The L-Phe concentration was determined via HPLC according to the instruction of Zorbax Eclipse-AAA columns on an Agilent 1100 HPLC. This column could achieve the resolution ratio at approximately 10 pmols. Derivatization was performed according to the manufacturer's protocol. L-Phe was separated by using mobile phase A $(40 \mathrm{mM}$ $\mathrm{Na}_{2} \mathrm{HPO}_{4} \mathrm{pH}$ 7.8) and phase $\mathrm{B}$ (Acetonitrile (ACN): $\mathrm{MeOH}: \mathrm{H}_{2} \mathrm{O}=45: 45: 10$ ) with gradient elution (The gradient variation ratio of phases $\mathrm{A}: \mathrm{B}$ with time was shown in Additional file 1: Table S2). Column temperature was set to $30{ }^{\circ} \mathrm{C}$ and the flow rate was $2 \mathrm{~mL} / \mathrm{min}$. One sample was quantitatively analyzed within $30 \mathrm{~min}$. All experiments were conducted in triplicate.

\section{Proteomic preparation and digestion}

Protein preparation and digestion were performed as follows: $5 \mathrm{~mL}$ of fermentation broth (at logarithmic early growth) was centrifuged for $20 \mathrm{~min}$ at $3500 \mathrm{~g}$ and $4{ }^{\circ} \mathrm{C}$. The supernatants were washed with PBS buffer thrice and immediately frozen with liquid nitrogen. Cell pellets were then resuspended in a UA buffer $(8 \mathrm{M}$ Urea dissolved in $0.1 \mathrm{M}$ Tris- $\mathrm{HCl}(\mathrm{pH} 8.5)$, containing $1 \% \beta$ mercaptoethanol $(\mathrm{v} / \mathrm{v})$, and $10 \mathrm{mM}$ DTT) and broken via sonication cracking on ice ( $5 \mathrm{~s}$ on, $5 \mathrm{~s}$ off for a total of $10 \mathrm{~min}$ ). The samples were centrifuged for $10 \mathrm{~min}$ at $2275 \mathrm{~g}$ and $4{ }^{\circ} \mathrm{C}$ and the resulting supernatant was filtered through a sterile membrane filter (Millipore, PES polymer cast, $0.22 \mu \mathrm{m})$. A protein pellet was quantitated using a 2D-Quant Kit (GE Healthcare) and dissolved in digestion buffer (100 mM TEAB (triethylammonium bicarbonate)) to a final concentration of $1 \mathrm{mg} / \mathrm{mL}$. Equal aliquots were digested with trypsin (Promega) overnight at $37^{\circ} \mathrm{C}$ and then lyophilized [20, 21].

\section{Proteomic data analysis}

Mass spectrum analysis was conducted via a Triple Time of Flight (TOF) 5600 mass spectrometer (AB SCIEX, USA). A NanoLC pre-column (Chromxp C18-LC-3 $\mu \mathrm{m}$, Eksigent) and an analytical column (C18-CL-120, Eksigent) were separately used to trap and elute peptides via gradient wash from 5 to 35\% Buffer B (Buffer A: 2\% ACN, $98 \% \mathrm{H}_{2} \mathrm{O}$, Buffer B: $98 \%$ ACN, $2 \% \mathrm{H}_{2} \mathrm{O}, 0.1 \%$ formic acid) at a flow rate of $300 \mathrm{~nL} / \mathrm{min}$. Full-scan MS was performed in the positive ion mode with a nano-ion spray voltage of $2.5 \mathrm{kV}$ from 350 to $1500(\mathrm{~m} / \mathrm{z})$, with up to 30 precursors selected for MS/MS if the precursors exceeded a threshold of 125 counts per second. Charged peptides ranging from +2 to +5 were screened for the MS/MS analysis. The collision energy (CE) for the collision-induced dissociation (CID) was automatically controlled using an Information-Dependent Acquisition (IDA) CE parameter script to achieve optimum fragmentation efficiency.

To identify the proteins within samples, theoretical peptide spectra of proteins deposited in a protein database were matched to the acquired tandem mass spectra using the search engine MASCOT. Then, the approach was termed spectral counting, implying counting and comparison of the number of fragment-ion spectra (MS/ MS) acquired for peptides of a special protein.

\section{Real-time PCR analysis}

Total RNA extraction was conducted via RNA prep pure Cell/Bacteria Kit (DP430, Tiangen, China) and the purified RNA was stored at $-80{ }^{\circ} \mathrm{C}$. cDNA synthesis was performed via PrimeScript ${ }^{\circ}$ 1st Strand cDNA Synthesis Kit (D6110A, TAKARA, Japan) and the purified cDNA was stored at $-20{ }^{\circ} \mathrm{C}$. Real-time PCR (RT-PCR) was performed with the ABI Prism7000 Sequence Detection System using the SYBR Green PCR Master Mix. The amplification conditions were as follows: $10 \mathrm{~min}$ at $95{ }^{\circ} \mathrm{C}$, and a two-step cycle at $95{ }^{\circ} \mathrm{C}$ for $15 \mathrm{~s}$ and $60{ }^{\circ} \mathrm{C}$ for $60 \mathrm{~s}$ for a total of 45 cycles (primers used are listed in Additional file 1 : Table S3). All samples were performed in triplicate. A non-template control reaction mixture was included for each gene as negative control. All data were normalized using the ihfB gene as an internal control (housekeeping gene) [22]. 


\section{Results}

Improving the available intracellular PEP by inactivating the PTS system

Previous research has shown that the glucose transportation and phosphorylation capabilities of the PTS system could be replaced by co-overexpressing alternative enzymes D-galactose transporter (GalP) and glucokinase (Glk) to allow higher PEP availability for L-Phe formation [23]. In this case, we attempted to reproduce this approach and determine the appropriate expression levels of both enzymes.

The Xllp02 strain was created by deleting the ptsH gene in Xllp01 strain. As expected, the cells growth of Xllp02 was dramatically decreased in shake flask fermentation due to the lack of sufficient glucose intake (Fig. 1a). A previous study [23] applied several artificial promoters exhibiting different strength for modulating the expression of galp and glk. Similarly, two promoters

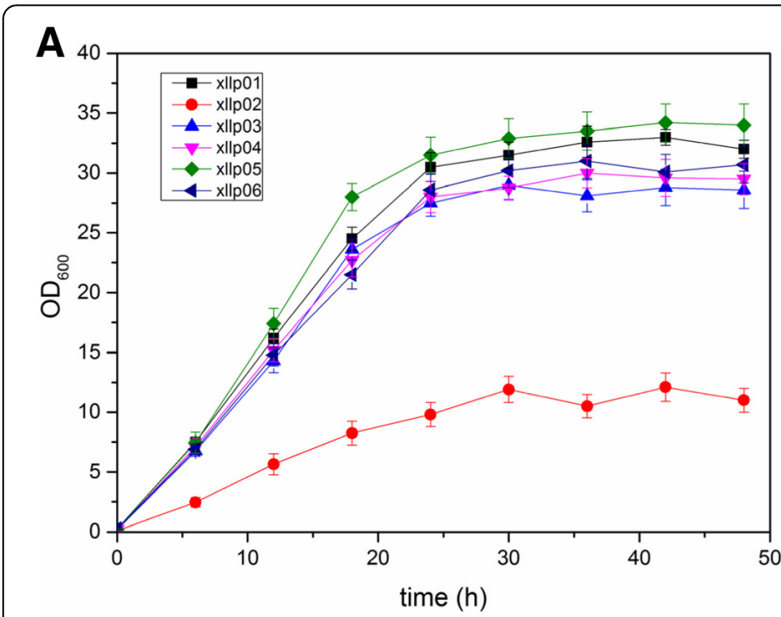

B

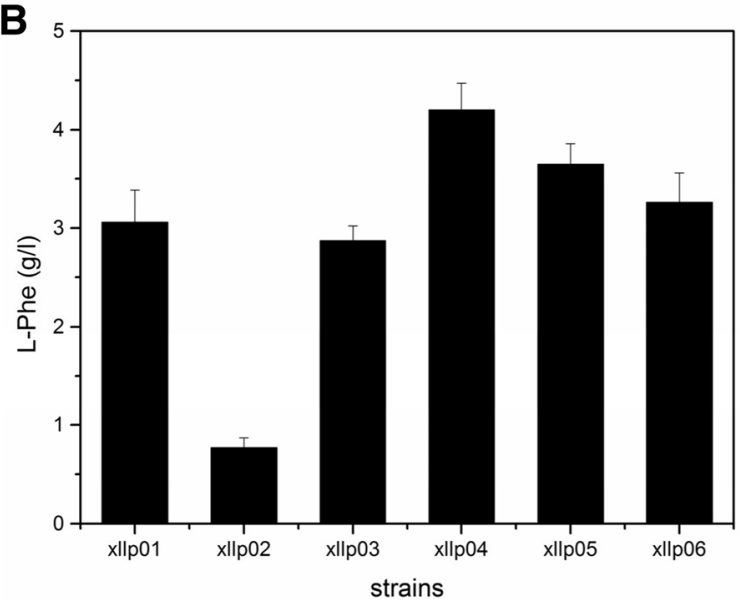

Fig. 1 Growth curves and fermentation results of the recombinant strains combinational modulating the galp and glk genes with different promoters. a Growth curves of the recombinant strains; $\mathbf{b}$ The fermentation results of the recombinant strains in shake flasks. The experiment was repeated three times, and measurements are represented as means with their standard deviation
Pm93 and Pm37 were selected to combinatorially modulate the expression of both genes thus maintaining the proper specific growth rate and glucose utilization of the recombinant strains. To enhance glucose uptake in Xllp02, two artificial promoters exhibiting different strength Pm37 and Pm93 [23] were selected to combinatorially modulate galP and glk gene expression in chromosome. Four engineered strains, in which galP and $g l k$ were separately controlled by Pm37 and Pm93 (Table 1), were constructed to test the growth curve, LPhe titer, and yield. Fig. 1a showed that the growth rate of four strains recovered at different degrees compared to the Xllp02 strain. Interestingly, a good positive correlation was observed between L-Phe production and the growth of strains. This may be due to the unanimous demand of the carbon resourc e for L-Phe production and cell growth. The highest titer of L-Phe was observed in the Xllp04 strain $(4.2 \mathrm{~g} / \mathrm{L})$, in which galP and $g l K$ were separately modulated by $\mathrm{m} 37$ and $\mathrm{m} 93$ (Fig. 1b).

\section{Engineering the HTH of TyrR to optimize L-Phe synthetic pathway}

Although the TyrR regulator has evolved a diverse range of specific regulations on the genes involved in L-Phe synthesis, it is difficult to relieve such inhibition by knocking out tyrR. Our results showed that the L-Phe production decreased by $32 \%$ when tyrR gene was knocked out in the Xllp04 strain (Additional file 1: Figure S1). To solve this problem, we attempted to engineer the HTH structure in the C-domain of the TyrR protein to relieve the TyrR's repression, as several enzymes were up-regulated in the tyrR mutation strains [13]. The mutation site was verified via PCR and sequence analysis (Additional file 1: Figure S2).

The replaced residues were located in the second $\alpha$ helix of the HTH motif, which might be expected to specifically interact with key bases in the TyrR boxes in the promoter region of the regulated genes. The amino acids at position 1, 2, and 6 had been suggested to be involved in specific contacts with the promoters. Therefore, we attempted to change these amino acids to observe activation or repression effects on the genes involved in the L-Phe synthetic pathway. Five potential site mutations were separately introduced into the HTH domain of the TyrR protein in the chromosome of Xllp04 strain, generating Xllp07 (S493 T), Xllp08 (T495I), Xllp09 (N499D), Xllp10 (A498V), and Xllp11 (S482 N), respectively. After fermentation, the five strains presented a similar trend of growth, but showed significant differences on the titer of L-Phe (Fig. 2a). The Xllp08 strain showed an apparent increase in L-Phe production and the titer reached $6.31 \mathrm{~g} / \mathrm{L}$, which was approximately 1.5-times of that of Xllp04. To investigate the hyperproducing mechanism of this strain, a quantitative PCR (qPCR) analysis was 

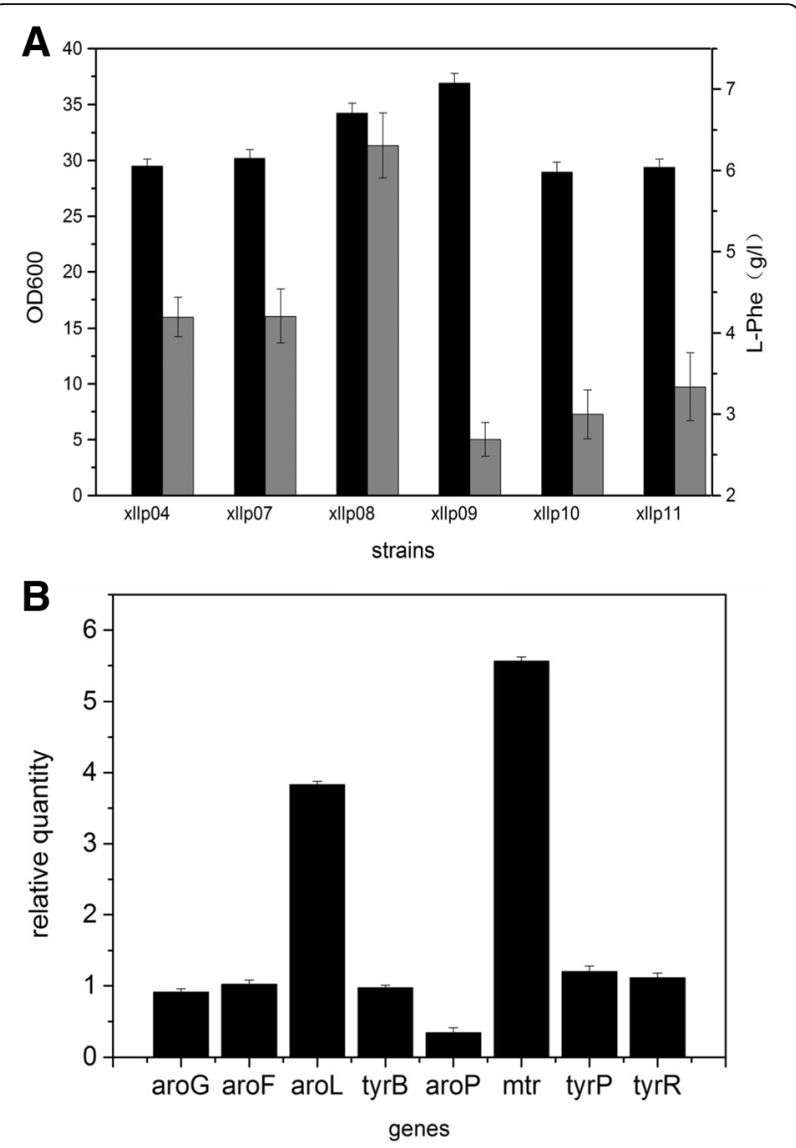

Fig. 2 Fermentation results and transcriptional analysis of the TyrR mutant strains. a Fermentation results of the TyrR mutant strains in shake flasks. Black columns stands for $\mathrm{OD}_{600}$ and gray columns stands for the L-Phe production. b Real-time PCR analysis consequences of the genes regulated by the TyrR protein in XIlp08 contrast to XIIp04. Experiments were conducted in triplicate

conducted to monitor differences in transcriptional level of genes regulated by TyrR protein between Xllp08 and Xllp04.

As shown in Fig. 2b, aroL (coding shikimate kinase), which was directly involved in the L-Phe metabolism was up-regulated 3.83-times in Xllp08. In addition, the expression of the $m t r$ increased 5.56-times. This could contribute to the enhancement of tryptophan uptake, thus reducing the requirement of tryptophan during the fermentation process [24]. The aromatic amino acid transporter aroP decreased 0.71-time in Xllp08. Other genes $(\operatorname{tyr} B$, aroG, tyrR, and $\operatorname{tyr} P$ ) did not significantly change in the transcriptional level between both strains.

\section{Enhancing the L-Phe synthetic pathway flux via proteo- mics analysis}

To determine the targets for further engineering of the Xllp08 strain, label-free proteomics was performed to investigate differences in protein expression profiles between the Xllp08 and the E. coli W3110 strain. Protein samples were collected during the early logarithmic growth and subjected to proteomic analysis. The strict confidence criteria for identification of a total of 1447 proteins were identified in this experiment and roughly represented $40 \%$ of the total predicted proteins in E. coli. W3110 (Fig. 3). Among the identified proteins, 720 proteins presented increased expression level, while the expression level of 705 proteins was decreased in the Xllp08 strain. In terms of the number of proteins that were identified in each functional category, the most frequently detected category was "Cellular protein metabolic process", supplying roughly $16 \%$ of the entire proteins identified in the proteomics analysis. By setting the criteria for a cutoff of $p$ value below 0.05 , we focused on the proteins involved in the synthesis of glucose to L-Phe. As shown in Fig. 4a, enzymes in the EMP and PP pathways were generally upregulated, while enzymes in the TCA cycle were downregulated in the Xllp08 strain. This indicated that sufficient supply of precursors (PEP and E4P) is of vital importance for increased L-Phe production. In contrast, the TCA cycle seemed virtually irrelevant to the biosynthesis of L-Phe in the Xllp08 strain, which agreed with a previous study [25]. We assumed that the lower expression level in the TCA cycle ensured that sufficient PEP could be channeled into L-Phe production. Therefore, we attempted to overexpress genes encoding PEP and E4P synthesis enzymes ( $t k t A, \operatorname{talB}, p c k A$, and pps $A)$ in Xllp08 to increase the accumulation of precursors of L-Phe. However, all of these recombinant strains showed a severe growth reduction without obvious increase in L-Phe production (Additional file 1: Figure S3). We assumed that carbon flux between the PP pathway and TCA cycle reached a relative balance for cell growth and L-Phe production in Xllp08, and overexpressing either of them would cause a severe disturbance in cells, which acted as a metabolic overload for L-Phe production.

In addition to AroF and PheA which were overexpressed in plasmid p15A [26], other enzymes in the SHIK pathway such as AroB, AroK, AroL, AroA, and TyrB were also significantly increased in Xllp08 (Fig. $4 b)$. Surprisingly, AroD (2.0-times), YdiB (1.3-times), and AroC (1.02-times) were not up-regulated as dramatically as other enzymes, suggesting that these steps may be bottlenecks in Xllp08. To confirm this, $\operatorname{aroD}, y d i B$, and aro $C$ were separately ligated in the pBR322, and transformed into the Xllp08 strain thus producing Xllp16, Xllp17, and Xllp18. The fermentation results of these strains are shown in Fig. 5. The L-Phe titers of Xllp17 $(3.67 \mathrm{~g} / \mathrm{L})$ and Xllp18 $(1.86 \mathrm{~g} / \mathrm{L})$ remained flat or falling compared to that of the Xllp08. In contrast, a prominent increase in L-Phe production was observed in Xllp16 $(7.43 \mathrm{~g} / \mathrm{L})$; however, the final OD was only $47.3 \%$ that of Xllp08. A trade-off exists between the accumulation of 


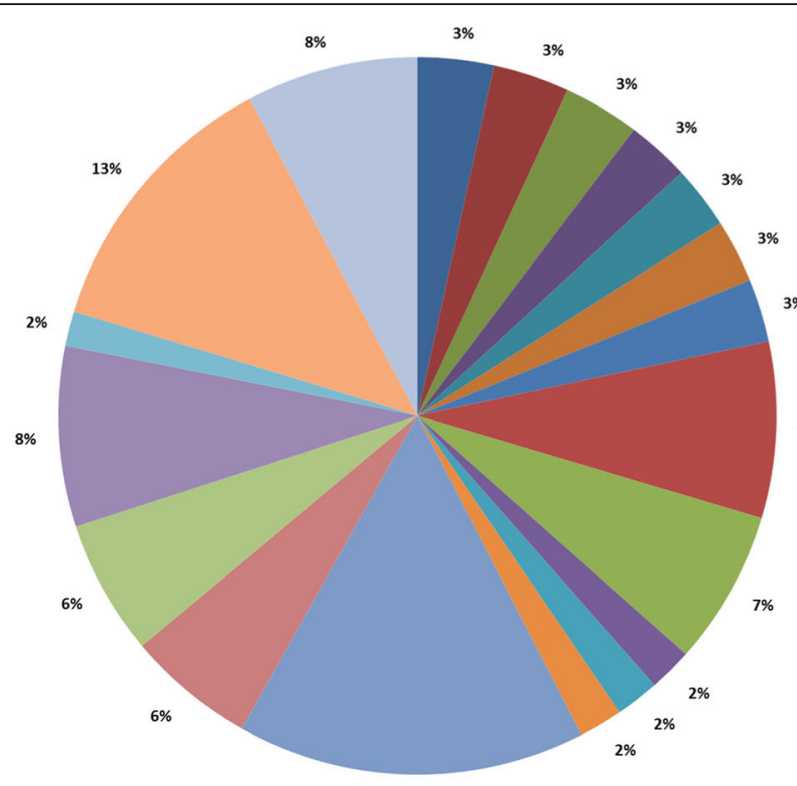

$16 \%$
- protein transport

- aminoglycan metabolic process

m establishment of protein localization

- cell wall macromolecule biosynthetic process

- peptidoglycan biosynthetic process

- cellular cell wall macromolecule metabolic

process

- aminoglycan biosynthetic process

n polysaccharide metabolic process

= polysaccharide biosynthetic process

- regulation of cell morphogenesis

- regulation of cell shape

regulation of anatomical structure

morphogenesis

= protein metabolic process

II lipid biosynthetic process

II cellular lipid metabolic process

= carbohydrate blosynthetic process

= cell redox homeostasis

- cellular protein metabolic process

nNA metabolic process

Fig. 3 Gene ontology analysis of the proteins identified by the proteomics
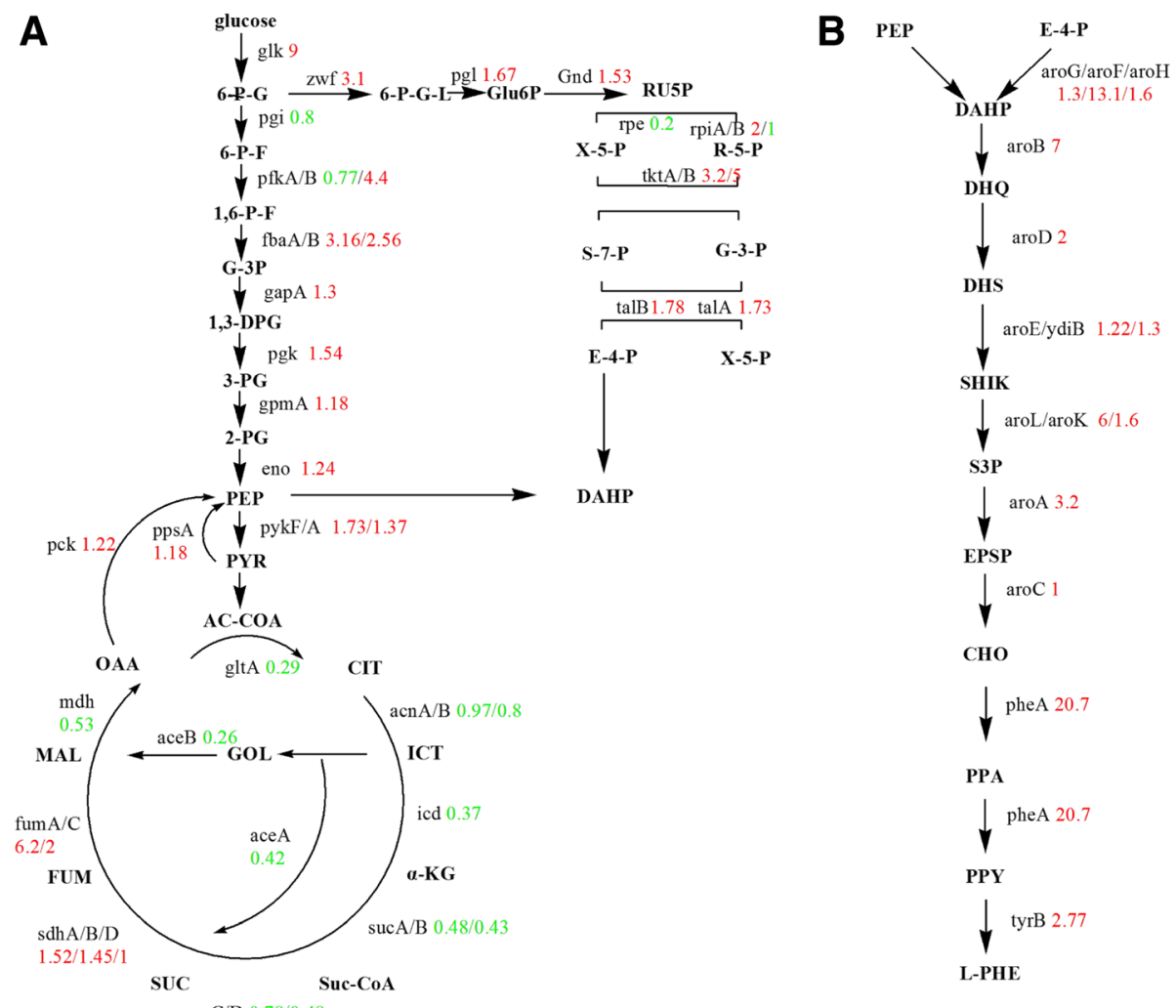

sucC/D $0.78 / 0.48$

Fig. 4 Proteomic analysis of the genes in XIlp08 and wild-type E. coli W3110. a Comparison of central carbon metabolism between wild-type W3110 and XIIp08. Two precursors (PEP and E4P) of the L-Phe synthesis were located in the EMP pathway and PP pathway respectively, the distribution of which would directly affect the L-Phe production and yield. The red data meant upregulation and green data meant downregulation. $\mathbf{b}$ The great disparity between two studied strains in the aromatic amino acid synthesis pathway. This figure showed comparion of enzymes in the L-Phe generating pathway between XIIp08 strain and wild-type E. coli W3110 


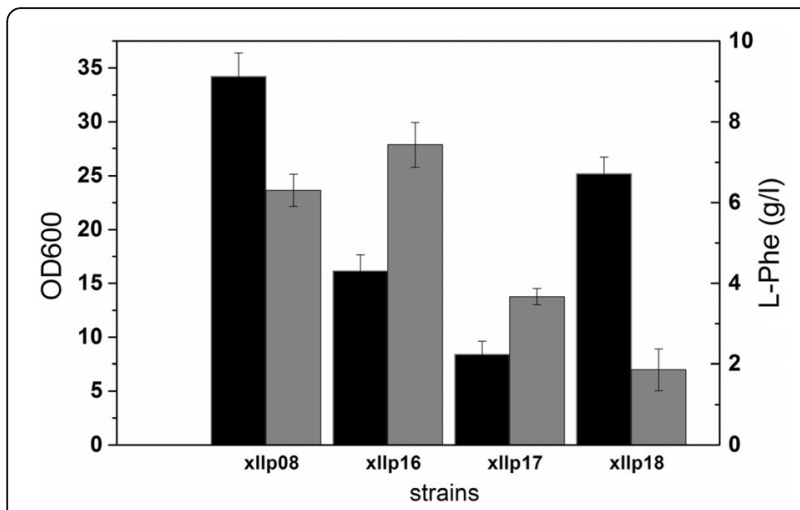

Fig. 5 Fermentation results of the XIIp08 strains and its derivative strains. XIIp16, XIlp17, and XIlp18 were obtained by overexpressing aroD, ydiB and aroC in Xllp08 strains, respcetively. The black column meant $\mathrm{OD}_{600}$ and the gray column meant L-Phe titer. Experiments were conducted in triplicate

biomass and the production of target compounds in industrial microorganisms [27]. Therefore, it was necessary to optimize the expression levels of aroD to further increase the L-Phe titer of Xllp08. To solve this problem, five promoters exhibiting different strength were chosen from the MIT parts registry (http://parts.igem.org/) to overexpress $a r o D$ in the Xllp08 strain. The relative strength of the selected promoters was given in Additional file 1: Figure S4. The final OD of the recombinant strains presented a decreasing trend accompanied with an increase of promoter strength, while the L-Phe production initially increased and remained stable with a further increase of aroD expression level (Fig. 6). In light of the biomass and the titer and productivity of L-Phe, the Xllp21 strain was chosen as the optimized strain for further engineering.

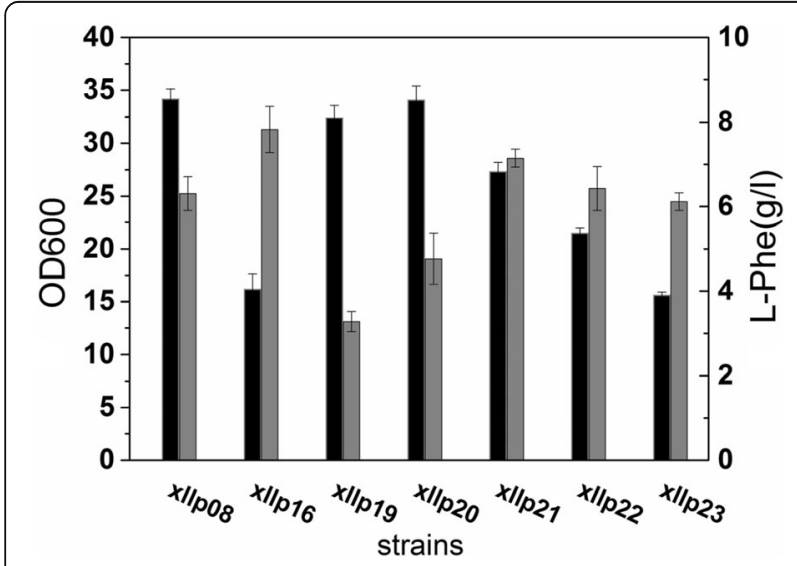

Fig. 6 Fermentation results of the recombinant strains overexpressing aroD gene with different promoters in Xllp08 strain. The black column meant $\mathrm{OD}_{600}$ and the gray column meant the L-Phe titer. The expreiments were repeated three times
Transcription analysis of the recombinant strain To observe the relevant genic expression disturbance via overexpression of aroD in Xllp21, a subset of 16 genes, including genes involved in L-Phe biosynthesis, glucose utilization, and L-Phe precursor metabolism were selected for analysis via RT-PCR [22, 28]. As shown in Fig. 7 , the most notable difference appears in the $p g i$ gene, with a 7.21-times increase in the Xllp21 strain compared with Xllp08. The $z w f$, $t k t A$ and $t a l B$ were also up-regulated 1.19-times, 1.17-times, and 1.66-times, respectively. This indicated that the metabolic flux of glycolysis and pentose phosphate pathway was enhanced. The icd gene showed no apparent change (0.97-time) while the ppsA gene coding for phosphoenolpyruvate synthase (which stood for gluconeogenesis pathway from malic acid (MAL) to pyruvic acid (PYR) and PEP) showed a 2.02-times increase. This would facilitate more PEP to pour into the L-Phe synthetic pathway and contribute to increasing the conversion ratio from glucose to L-Phe [29]. Therefore, it can be concluded that the Xllp21 strain was engineered to channel more carbon influx from the central metabolism towards the shikimate pathway by enhancing EMP and PP pathway, while converting flux in the TCA cycle into PEP.

Compared to Xllp08, the transcriptional level of genes tyrB and $y d i B$ were significantly increased in Xllp21. Other genes of the L-Phe synthetic pathway, such as aro $C$, aroB, aroK, aroE, and pheA showed no apparent differences between both strains. In contrast, aro $L$ and aro $A$ genes showed a 0.61-time and a 0.28-time decrease, respectively. We suggest that the downregulation of $\operatorname{aro} A$ was due to the lack of sufficient intracellular PEP, which is necessary for this reaction catalyzed by AroA. In light of this, further study should be conducted

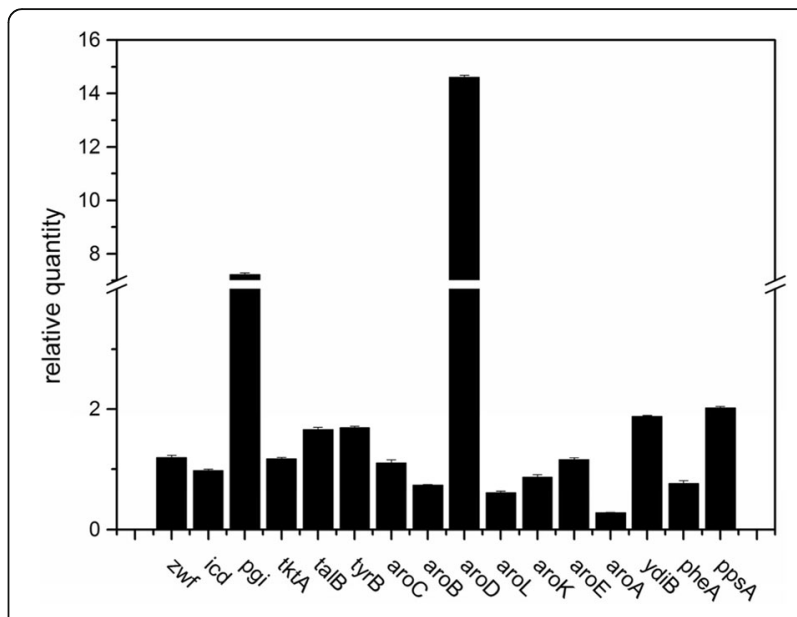

Fig. 7 Relative transcription level of genes in XIIp21 and XIIp08 by real-time PCR analysis. Experiments were conducted in triplicate, and measurements are represented as means with their standard deviation 
on the fine-tuning of the entire enzymes in the L-Phe synthetic pathway.

\section{L-Phe production in a $5 \mathrm{~L}$ fermenter}

We performed a fed-batch culture with intermittent glucose feeding to investigate the production of L-Phe by the Xllp21 strain (Fig. 8, Additional file 2). Compared to the original strain Xllp01, the final biomass of Xllp21 showed no significant difference. However, the glucose consumption rate of Xllp21 in the lag phase was much faster than that of Xllp01, which indicates to contribute to L-Phe accumulation. For Xllp21, L-Phe started to accumulate in the exponential growth phase after $18 \mathrm{~h}$ of cultivation, which is $4 \mathrm{~h}$ earlier compared to Xllp01. The final L-Phe production of Xllp21 was $72.9 \mathrm{~g} / \mathrm{L}$ at $52 \mathrm{~h}$, with a productivity of $0.26 \mathrm{~g} / \mathrm{g}$ glucose. Plasmid stability was assessed after fermentation by testing the resistance to the ampicillin antibiotic marker. More than $96 \%$ of the cells retained ampicillin resistance, indicating that the plasmid p-j23106-arod remained stable throughout cultivation. Compared to the parent strain Xllp01 (Fig. 8a), the L-Phe titer was improved by $62 \%$. Moreover, the yield of glucose was also greatly improved by $39.7 \%$ compared to the parent strain $(0.186 \mathrm{~g} / \mathrm{g}$ glucose $)$.

\section{Discussion}

In order to facilitate the comparison with other strains, we summarized all fermentation values (including OD, L-Phe titer and yield on glucose) of all recombinant strains in this study in Table 2.Through the system level engineering, we obtained a high L-Phe producer Xllp21, which was capable of producing $72.6 \mathrm{~g} / \mathrm{L}$ L-Phe with a yield of $0.26 \mathrm{~g} / \mathrm{g}$ glucose under the non-optimized fermentation condition. As far as we know, the titer of Xllp21 has exceeded the highest titer of L-Phe reported
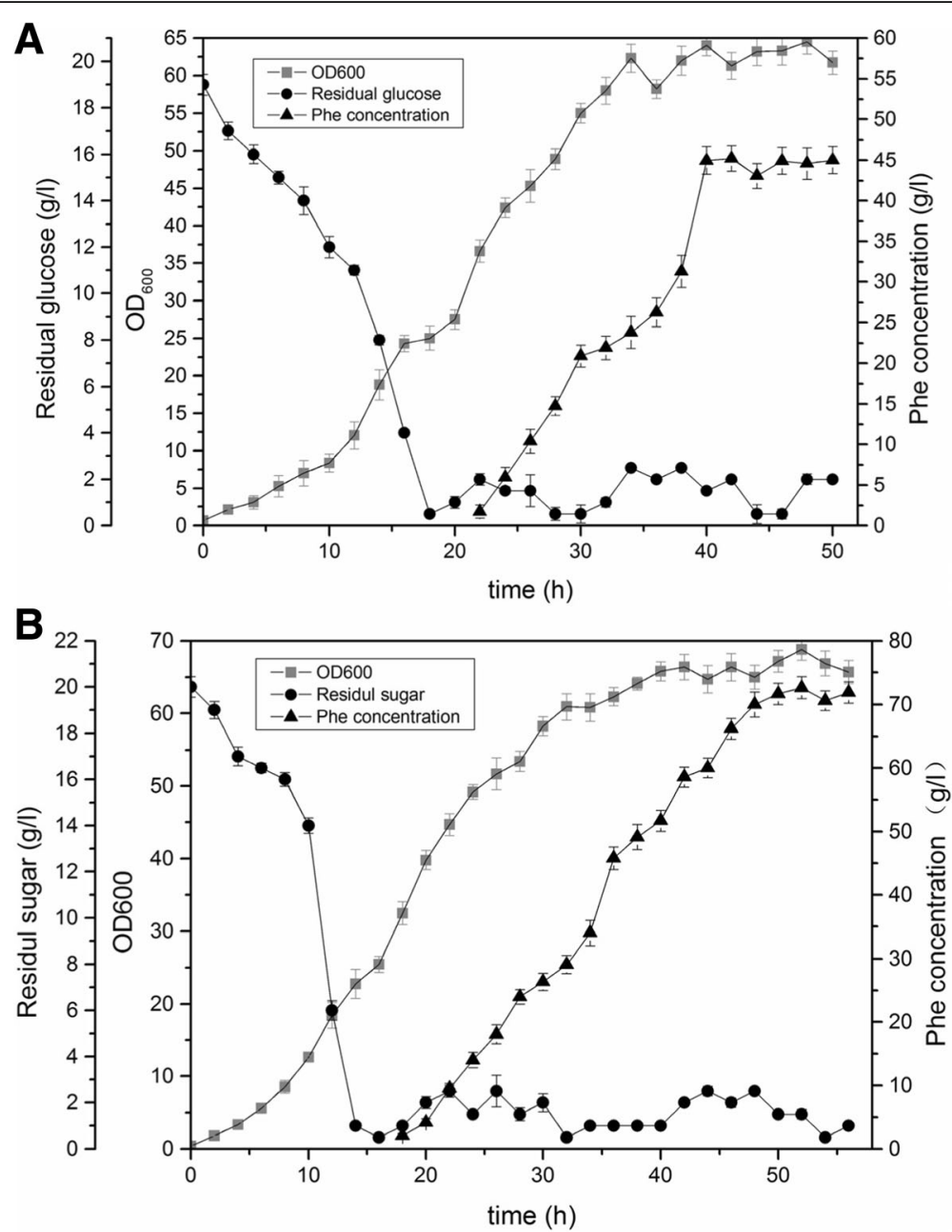

Fig. 8 Time course of L-Phe production by strain XIIp01 (a) and XIIp21 (b) in a 5 L fermenter. Dots meant residual glucose concentration, blocks meant the $\mathrm{OD}_{600}$ and triangles meant the L-Phe concentration. The final L-Phe yield and titer was basically constant among the three replicates and one of the three times was given as an example to show the fermentation results 
Table 2 Fermentation values of the recombinat strains

\begin{tabular}{|c|c|c|c|}
\hline Strains & $\begin{array}{l}\text { Titer of } \\
\text { Phenylalanine }(\mathrm{g} / \mathrm{L})\end{array}$ & $\mathrm{OD}_{600}$ & $\begin{array}{l}\text { Yield } \\
\text { (to glucose } \mathrm{g} / \mathrm{g} \text { ) }\end{array}$ \\
\hline XIlp01 & $3.06(45)$ & 32.5 & $0.07(0.18)$ \\
\hline XIlp02 & 0.77 & 11.6 & 0.04 \\
\hline XIlp03 & 2.87 & 28.7 & 0.06 \\
\hline XIlp04 & 4.2 & 29.6 & 0.09 \\
\hline XIlp05 & 3.65 & 34.1 & 0.08 \\
\hline XIlp06 & 3.26 & 30.3 & 0.07 \\
\hline XIIp07 & 4.21 & 30.2 & 0.09 \\
\hline XIIp08 & 6.31 & 34.2 & 0.12 \\
\hline Xllp09 & 2.69 & 36.9 & 0.06 \\
\hline XIIp10 & 2.98 & 28.9 & 0.07 \\
\hline XIIp11 & 3.34 & 29.4 & 0.07 \\
\hline XIIp12 & 1.18 & 15.9 & 0.05 \\
\hline XIIp13 & 3.69 & 14.1 & 0.08 \\
\hline Xllp14 & 1.74 & 15.3 & 0.05 \\
\hline XIIp15 & 1.68 & 25.9 & 0.04 \\
\hline XIIp16 & 7.43 & 16.2 & 0.15 \\
\hline XIIp17 & 3.67 & 8.4 & 0.08 \\
\hline XIIp18 & 1.86 & 25.2 & 0.04 \\
\hline XIIp19 & 3.28 & 32.4 & 0.07 \\
\hline XIIp20 & 4.77 & 34.1 & 0.11 \\
\hline XIlp21 & 7.15 (72.6) & 27.3 & $0.16(0.26)$ \\
\hline XIlp22 & 6.43 & 21.5 & 0.14 \\
\hline XIIp23 & 6.12 & 15.6 & 0.13 \\
\hline
\end{tabular}

Note: All datas were colleted from the shake flask fermentation and calcuated by triplicate samples. Data in the parenthesis was colleted from a 5-L fermenter

previously (57.63 g/L [8]) and was comparable to the highest yield $(0.27 \mathrm{~mol} / \mathrm{mol}$ glucose [30]) reported.

In a PTS $^{-}$strain, the uptake and phosphorylation of glucose could be achieved by combinatorially overexpressing the galP and glk. A previous study [23] provided several artificial promoters for galp and glk modulation exhibiting at different strength. By applying this strategy, a high glucose uptake rate was obtained during the fedbatch fermentation of the Xllp04 strain (Fig. 1a). In light of the industrial production process, we avoided to use a weak promoter because the low expression level of galP and $g l k$ would hamper glucose uptake, resulting in a low specific growth rate. In addition, it was found that the expression level of galP was predominant for effective glucose uptake in our industrial strain because the final $\mathrm{OD}_{600}$ of the strains increased accompanied by an increase of the promoter strength of galP. However, higher cell growth did not lead to a higher L-Phe titer. The fermentation result (Fig. 1b) showed that a combinatorially expressing of galP and glk by using $\mathrm{m} 37$ and $\mathrm{m} 93$ resulted in the highest L-Phe titer, which was higher than the production of Xllp06 using both strong promoters to control galP and glk. This indicated that the expression levels of these two genes should be finetuned to reach optimal glucose utilization.

In $E$. coli, the synthesis and transport of aromatic amino acids are regulated by the transcription factor TyrR. The C-terminal region of the TyrR protein contains a DNA-binding domain, allowing it to bind specific TyrR box sequences in regulated genes. Previous research showed that the activity of enzymes that are involved in aromatic amino acid biosynthesis altered when mutagenesis was introduced into the C-terminal HTH motif of TyrR [16]. Here, we selected five potential mutants that may deregulate the inhibition effects of aroG, $\operatorname{tyr} B$, and aroL. Interestingly, the five mutant strains exhibited distinct trend of cell growth and L-Phe production, indicating the mechanism of TyrR regulation in $E$. coli to be far more complex than expected. It is likely that the site mutation in the HTH motif might have influenced the interaction with other binding sites associated with unknown genes, which were responsible for cell growth. Transcriptional analysis of Xllp08 showed that this mutation (T495I) caused an increase in the transcriptional level of the $m t r$ and aroL. It is possible that the mutant on the HTH motif changed the affinity between TyrR regulator and the TyrR box on the promoters of these genes, which led to effective binding of the RNA polymerase to the $\mathrm{P}_{\mathrm{mtr}}$ and $\mathrm{P}_{\mathrm{aroL}}$, thus enhancing the transcription level of these genes. Further research is required to reveal the mechanism between TyrR and nucleic acid through which application of this regulator can be improved.

Due to the significant role of PEP, many manipulations of the ppsA gene have been conducted to improve the enzymatic properties [22, 23]. However, overexpressing the ppsA gene in Xllp08 failed to improve the production of L-Phe due to metabolic overload. Interestingly, the aroD overexpressing strain showed an additional pps A up-regulation. We assumed that a great demand for a certain metabolite could in turn accelerate its biosynthesis. Furthermore, another potential strategy for improving available intracellular PEP was to inactivate the PEP-consuming enzymes PykA or PykF. This strategy had been applied to enhance the additional availability of PEP for the synthesis of shikimic acid in E. coli [31]. Strains with deleted $p y k A$ and $p y k F$ could obtain higher concentrations of intracellular PEP to be channeled into the L-Phe synthetic pathway.

It was necessary to add antibiotics in the feed-batch fermentation. In this study, $100 \mu \mathrm{g} / \mathrm{mL}$ ampicillin was added in the initial of fermentation in a $5 \mathrm{~L}$ fermentor. Given that the ampicillin antibiotic doesn't work for more than $16 \mathrm{~h}$, we replenished the ampicillin antibiotic 
after $16 \mathrm{~h}$ of fermentation to prevent the bacterial contamination and to maintain the plasmid stability. After that it was not necessary to add ampicillin antibiotic into the fermentor because our engineered strain was so competitive that it was almost impossible to result in bacterial contamination.

As to the resistance loss, we assumed that the optical density of cells were so high that $100 \mu \mathrm{g} / \mathrm{mL}$ ampicillin antibiotic couldn't sufficiently maintain all cells' plasmid stability (In fact, the final OD of strains could reach to $70 \sim 75$ in a $5 \mathrm{~L}$ fermentor). However, overdose of ampicillin will bring damage to the physiology of cells, which will bring about the productivity loss. On the whole, we determined to add $100 \mu \mathrm{g} / \mathrm{mL}$ ampicillin antibiotic into the medium, although $4 \%$ of the cells in the $5 \mathrm{~L}$ fermenter was found to lose the ampicillin resistance at the end of the fermentation, the final titer and yield of L-Phe was not obviously affected by the plasmid loss of a small number of producers.

\section{Conclusions}

In this study, the glucose utilization system was reconstructed by modulating the galp and glk genes in a PTS strain to decrease the PEP consumption. The TyrR regulator was also investigated to modify the transcriptional inhibition in the aromatic amino acid metabolism. The T495I mutation introduced in the DNA binding domain of TyrR greatly altered binding the aroL and mtr genes, thus intensifying the synthetic pathway and reducing the by-product. Furthermore, the protein expression in the central metabolism and the L-Phe synthetic pathway were observed via proteomic analysis. This study showed that the third step of the SHIK pathway was a bottleneck step for L-Phe production in our recombinant strain. The finetuned overexpression of the enzyme AroD improved the glucose uptake rate and gluconeogenesis pathway, thus increasing the production of L-Phe in an already advanced production strain. The detailed characterization presented here provides a systematic approach for designing an engineered strain capable of producing L-Phe, which will be useful for further metabolic engineering.

\section{Additional files}

Additional file 1: Table S1. Primers used in the genetic engineering. Table S2. The gradient variation ratio of phases A:B in HPLC analysis of L-Phe concentration. Table S3. Primers used in the Real-time PCR analysis, Figure S1. Fermentation consequence of the tyrR knocked out strain. The gray column means $\mathrm{OD}_{600}$ and the black column means L-Phe titer Figure S2. Sequence alignment results of the TyrR $R_{w t}$ and TyrR mut This result was obtained by DNAMAN software. Figure S3. Overexpression of the precursors' synthesis encoded genes. The gray column means $\mathrm{OD}_{600}$ and the black column means L-Phe titer. Figure S4. Relative strength of five promoters that were used to overexpress AroD in Xllp08. (DOCX $582 \mathrm{~kb}$ )

Additional file 2. Online data of the fermentation of strain XIIp01 and Xllp21. (XLSX $431 \mathrm{~kb})$

\section{Acknowledgements}

Not applicable.

\section{Funding}

Nature Science Foundation of Tianjin City (CN) (16JCYBJC23500), Tianjin science and technology project (15PTCYSY00020), The Key Projects in the Tianjin Science \& Technology Pillar Program (14ZCZDSY00058), and Key Laboratory of Systems Microbial Biotechnology, Tianjin Institute of Industrial Biotechnology, Chinese Academy of Sciences. All the fundings mentioned above have no roles in the design of the study and collection, analysis, and interpretation of data and in writing the manuscript.

\section{Availability of data and materials}

All data generated or analysed during this study are included in this published article and its supplementary information files.

\section{Authors' contributions}

YFL, YRX and DZ conceived of the manuscript. YFL and YRX performed the experiment. YFL, YRX, DQD, JPW, BWZ and DZ analysed data and wrote the manuscript. All authors have read and approved the final manuscript.

Ethics approval and consent to participate

Not applicable.

Consent for publication

Not applicable.

Competing interests

The authors declare that they have no competing interests

\section{Publisher's Note}

Springer Nature remains neutral with regard to jurisdictional claims in published maps and institutional affiliations.

\section{Author details}

'Tianjin Institutes of Industrial Biotechnology, Chinese Academy of Sciences, Tianjin 300308, People's Republic of China. ${ }^{2}$ Department of Biological Engineering, School of Chemical Engineering and Technology, Tianjin University, Tianjin 300072, People's Republic of China. ${ }^{3}$ Key Laboratory of Systems Microbial Biotechnology, Chinese Academy of Sciences, Tianjin 300308, People's Republic of China. ${ }^{4}$ School of Food Science and Technology, National Engineering Research Center of Seafood, Dalian Polytechnic University, Dalian 116034, People's Republic of China.

Received: 10 May 2017 Accepted: 18 January 2018

Published online: 30 January 2018

\section{References}

1. Wendisch VF, Bott M, Eikmanns BJ. Metabolic engineering of Escherichia Coli and Corynebacterium glutamicum for biotechnological production of organic acids and amino acids. Curr Opin Microbiol. 2006;9:268-74.

2. Bongaerts J, Kramer M, Muller U, Raeven L, Wubbolts M. Metabolic engineering for microbial production of aromatic amino acids and derived compounds. Metab Eng. 2001;3:289-300.

3. Rodriguez A, Martinez JA, Flores N, Escalante A, Gosset G, Bolivar F. Engineering Escherichia Coli to overproduce aromatic amino acids and derived compounds. Microb Cell Factories. 2014;13(1):126.

4. Khamduang M, Packdibamrung K, Chutmanop J, Chisti Y, Srinophakun P. Production of I-phenylalanine from glycerol by a recombinant Escherichia Coli. J Ind Microbiol Biotechnol. 2009;36:1267-74.

5. Galvao TC, de Lorenzo V. Transcriptional regulators a la carte: engineering new effector specificities in bacterial regulatory proteins. Curr Opin Biotechnol. 2006;17:34-42.

6. Zhang F, Ouellet M, Batth TS, Adams PD, Petzold CJ, Mukhopadhyay A, Keasling JD. Enhancing fatty acid production by the expression of the regulatory transcription factor FadR. Metab Eng. 2012;14:653-60.

7. Ikeda M, Ozaki A, Katsumata R. Phenylalanine production by metabolically engineered Corynebacterium-Glutamicum with the Phea gene of Escherichia-Coli. Appl Microbiol Biotechnol. 1993;39:318-23. 
8. Liu SP, Liu RX, Xiao MR, Zhang L, Ding ZY, Gu ZH, Shi GY. A systems level engineered $\mathrm{E}$. Coli capable of efficiently producing L-phenylalanine. Process Biochem. 2014;49:751-7.

9. Chandran SS, Yi J, Draths KM, von Daeniken R, Weber W, Frost JW Phosphoenolpyruvate availability and the biosynthesis of shikimic acid. Biotechnol Prog. 2003:19:808-14.

10. Meza E, Becker J, Bolivar F, Gosset G, Wittmann C. Consequences of phosphoenolpyruvate: sugar phosphotranferase system and pyruvate kinase isozymes inactivation in central carbon metabolism flux distribution in Escherichia Coli. Microb Cell Factories. 2012;11(1):1-13.

11. Ray JM, Yanofsky C, Bauerle R. Mutational analysis of the catalytic and feedback sites of the tryptophan-sensitive 3-Deoxy-D-ArabinoHeptulosonate-7-phosphate synthase of Escherichia-Coli. J Bacteriol. 1988; 170:5500-6.

12. Zhou HY, Liao XY, Liu L, Wang TW, Du GC, Chen J. Enhanced Lphenylalanine production by recombinant Escherichia Coli BR-42 (pAP-B03) resistant to bacteriophage BP-1 via a two-stage feeding approach. J Ind Microbiol Biotechnol. 2011;38:1219-27.

13. Wang YJ, Zhao S, Somerville RL, Jardetzky O. Solution structure of the DNA-binding domain of the TyrR protein of Haemophilus influenzae. Protein Sci. 2001:10:592-8.

14. Pittard J, Camakaris H, Yang J. The TyrR regulon. Mol Microbiol. 2005;55:16-26.

15. Yang J, Ogawa Y, Camakaris H, Shimada T, Ishihama A, Pittard AJ. folA, a new member of the TyrR regulon in Escherichia Coli K-12. J Bacteriol. 2007; 189:6080-4.

16. Yang J, Ganesan S, Sarsero J, Pittard AJ. A genetic-analysis of various functions of the Tyrr protein of Escherichia-Coli. J Bacteriol. 1993;175:1767-76.

17. Ding D, Liu Y, Xu Y, Zheng P, Li H, Zhang D, Sun J. Improving the production of L-phenylalanine by identifying key enzymes through multi-enzyme reaction system in vitro. Sci Rep. 2016;6:32208.

18. Datsenko KA, Wanner BL. One-step inactivation of chromosomal genes in Escherichia Coli K-12 using PCR products. Proc Natl Acad Sci U S A. 2000;97: 6640-5.

19. Philippe N, Alcaraz JP, Coursange E, Geiselmann J, Schneider D. Improvement of pCVD442, a suicide plasmid for gene allele exchange in bacteria. Plasmid. 2004;51:246-55.

20. Megger DA, Bracht T, Meyer HE, Sitek B. Label-free quantification in clinical proteomics. Biochim Biophys Acta. 2013;1834:1581-90.

21. Qiao J, Huang S, Te R, Wang J, Chen L, Zhang W. Integrated proteomic and transcriptomic analysis reveals novel genes and regulatory mechanisms involved in salt stress responses in Synechocystis sp. PCC 6803. Appl Microbiol Biotechnol. 2013;97:8253-64.

22. Flores N, Flores S, Escalante A, de Anda R, Leal L, Malpica R, Georgellis D, Gosset G, Bolivar F. Adaptation for fast growth on glucose by differential expression of central carbon metabolism and gal regulon genes in an Escherichia Coli strain lacking the phosphoenolpyruvate:carbohydrate phosphotransferase system. Metab Eng. 2005;7:70-87.

23. Lu J, Tang J, Liu Y, Zhu X, Zhang T, Zhang X. Combinatorial modulation of galP and glk gene expression for improved alternative glucose utilization. Appl Microbiol Biotechnol. 2012;93:2455-62.

24. Heatwole VM, Somerville RL. The tryptophan-specific Permease gene, Mtr, is differentially regulated by the tryptophan and tyrosine repressors in Escherichia-Coli K-12. J Bacteriol. 1991;173:3601-4.

25. Chen L, Zeng AP. Rational design and metabolic analysis of Escherichia Coli for effective production of L-tryptophan at high concentration. App Microbiol Biotechnol. 2017;101:559-68.

26. Seizer G, Som T, Itoh T, J-i T. The origin of replication of plasmid p15A and comparative studies on the nucleotide sequences around the origin of related plasmids. Cell. 1983;32(1):119.

27. Liu Y, Zhuang Y, Ding D, Xu Y, Sun J, Zhang D. Biosensor-based evolution and elucidation of a biosynthetic pathway in Escherichia Coli. ACS Synth Biol. 2017;6(5):837-848.

28. Polen T, Kramer M, Bongaerts J, Wubbolts M, Wendisch VF. The global gene expression response of Escherichia Coli to L-phenylalanine. J Biotechnol. 2005;115:221-37.

29. Zhang X, Jantama K, Moore JC, Jarboe LR, Shanmugam KT, Ingram LO Metabolic evolution of energy-conserving pathways for succinate production in Escherichia Coli. Proc Natl Acad Sci U S A. 2009;106:20180-5.
30. Zhou H, Liao X, Wang T, Du G, Chen J. Enhanced I-phenylalanine biosynthesis by co-expression of pheA(fbr) and aroF(wt). Bioresour Technol. 2010;101:4151-6.

31. Escalante A, Calderon R, Valdivia A, de Anda R, Hernandez G, Ramirez OT, Gosset G, Bolivar F. Metabolic engineering for the production of shikimic acid in an evolved Escherichia Coli strain lacking the phosphoenolpyruvate: carbohydrate phosphotransferase system. Microb Cell Factories. 2010;9(1):1-12.

\section{Submit your next manuscript to BioMed Central and we will help you at every step:}

- We accept pre-submission inquiries

- Our selector tool helps you to find the most relevant journal

- We provide round the clock customer support

- Convenient online submission

- Thorough peer review

- Inclusion in PubMed and all major indexing services

- Maximum visibility for your research

Submit your manuscript at www.biomedcentral.com/submit
Biomed Central 